\title{
Colonic Perforation: A Medical Complication
}

Christopher Parsons, Elizabeth Chan, Richard P. T. Evans, Moustafa Mabrouk Mourad, Edmund Leung

Wye Valley NHS Trust, Hereford, United Kingdom

Received June 17, 2017; Accepted August 28, 2017.

Key words: Hypothyroidism - Sigmoid perforation - Constipation - Thyroid Hartmann's procedure

Abstract: Hypothyroidism is a common comorbidity that on acute presentation is often overlooked. It can be an easily managed condition; however non-compliance can have severe consequences. In the presented case it was requirement for emergency surgery that resulted in stoma formation. This case is a first example of the need to include patient's decision making process with regards to medication adherence in the setting of chronic disease.

Mailing Address: Edmund Leung, MD., FRCS, Department of Surgery, Wye Valley NHS Trust, Hereford, HR1 2ER, United Kingdom; Phone: +44(0) 143235 5444/5653; Fax: +44(0) 143236 4102; e-mail: Edmund.Leung@Wvt.nhs.uk 


\section{Introduction}

The Hartmann's procedure is the removal of the rectosigmoid colon with the formation of a rectal stump. This procedure is most commonly performed as emergency surgery for acute peritonitis, roughly $77 \%$ of cases (Pares et al., 2005). Other conditions that may require a Hartmann's procedure are intestinal obstruction (of which the most common cause is malignancy) and gastrointestinal bleeding. This procedure is reserved for critically unwell patients, as the gold standard operation would be anastomosis with diverting ileostomy (Liu et al., 2011).

Subclinical hypothyroidism occurs in roughly $3 \%$ of men and $8 \%$ of women (Chistiakov, 2005). Hypothyroidism is a condition where there is a lack of free thyroid hormones which are important biochemical modulators of metabolism. They have effects on the majority of body systems including vital actions like protein synthesis and regulating sympathetic activity. Clinical presentation of hypothyroidism includes bradycardia, drowsiness, hypothermia, congestive cardiac failure, immobile bowel and ileus (Canaris et al., 2000).

Hypothyroidism is known to cause gastrointestinal dysmotility however this is commonly unrecognised. Hypomotility, atony, pseudo-obstruction and in extreme cases even perforation has arisen as the result of hypothyroidism (Bergeron et al., 1997; Zachariah and Raja, 2010). The Association of Clinical Biochemistry, the British Thyroid Association and the British Thyroid Foundation state the incidence of hypothyroidism is around $1-2 \%$ demonstrating that although significant bowel complications are very rare there is a large proportion of the population at risk. We discuss a rare case of hypothyroidism causing a significant surgical emergency.

\section{Case report}

A 52-year-old lady with a background of acquired primary hypothyroidism secondary to thyroid malignancy, previous cholecystectomy and asthma who denied taking any regular medications presented with a fresh bleeding per rectum and lower abdominal pain. On arrival to the emergency department the patient was hypotensive, hypothermic and bradycardic. The patient lower abdominal tenderness and rectal examination revealed fresh blood. She was initially managed with analgesia, intravenous fluid resuscitation, intravenous antibiotics and tranexamic acid. Admission bloods were as follows: haemoglobin 117, white blood count 7.0, platelet 205, urea 6.9, estimated glomerular filtration rate 48, creatinine 105 (C-reactive protein not performed). She underwent an urgent CT (computed tomography) with intravenous contrast which identified moderate pneumoperitoneum extending from the pelvis closely associated to an inflamed sigmoid colon. No significant evidence of diverticulitis or malignancy was seen on the CT. Marked faecal loading was demonstrated throughout the colon and rectum. There were also changes congruent with sigmoid and rectal colitis with no obvious 

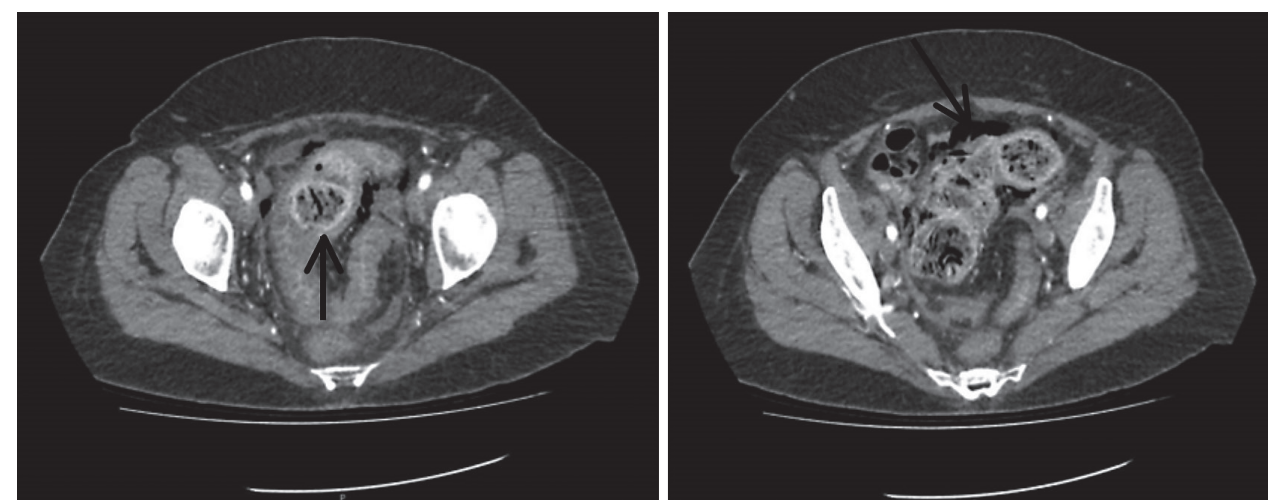

Figure 1 -Abdominal computed tomography scan shows pneumoperitoneum with enhancement of the distal sigmoid and rectum with faecal impaction.

site of mesenteric bleeding (Figure 1). The working diagnosis prior to theatre was of a stercoral perforation. The patient subsequently underwent an emergency laparotomy.

In keeping with the CT findings the patient had a sigmoid colon perforation with stercoral diffuse peritonitis with no other evident precipitating factors other than constipation. Therefore a Hartmann's procedure was performed which was uncomplicated. Two intra-abdominal surgical drains were placed and remained in-situ on transfer to the intensive therapy unit (ITU) for post-operative care. Post-operative bloods were performed in ITU which identified that the patient was markedly hypothyroid. Thyroid stimulating hormone (TSH) $>100 \mathrm{mU} / \mathrm{l}$ and free thyroxine (T4) $<0.3 \mathrm{pmol} / \mathrm{l}$. Treatment with IV tri-iodothyronine (T3) and IV antibiotics as per local antibiotic guidelines for intra-abdominal sepsis was given.

Prior to admission the patient had been taking no regular medication including thyroxine. She had not opened her bowels for 14 days and had not sought any medical help until the point of admission. After three days the patient was discharged from ITU and the intra-abdominal drains were removed. With specialist input from the endocrine team the intravenous thyroxine replacement was converted to oral levothyroxine. Inflammatory markers improved and she was switched to oral antibiotics and learnt to manage her own stoma, which started to function well. Histological analysis of the resected sigmoid did not reveal any evidence of malignancy, diverticulosis or colitis. During admission, other contributing factors for her constipation were excluded and the only abnormality identified was her marked hypothyroidism. She was counselled appropriately about compliance and was seen as an outpatient by the endocrine team. The patient declined stoma reversal during her routine surgical follow up. 


\section{Discussion}

Acquired primary hypothyroidism is a recognised consequence of thyroidectomy for malignancy that is easily managed by the consumption of oral thyroxine. In this case however, poor compliance to this treatment strategy resulted in severe hypothyroidism. It is clearly documented that decreased levels of thyroxine cause a proportional reduction in basal metabolic rate, which in turn reduces gut motility and causes constipation (Müller-Lissner et al., 2005; Ebert, 2010). Severe hypothyroidism and associated constipation has rarely been associated with surgical intervention (Bergeron et al., 1997).

Current literature has rarely demonstrated hypothyroidism causing significant surgical complications however this case adds to the cases as set forward by Bergeron et al. (1997), and Zachariah and Raja (2010). Bergeron et al. (1997) demonstrated 6 cases of hypothyroidism causing gastrointestinal complications including 1 case of gastrointestinal perforation (site unidentified at laparotomy), and Zachariah and Raja (2010) demonstrated a recto-sigmoid perforation in a patient with significant hypothyroidism. Perforation is a result of severe constipation is multi-factorial; however, the impact of hypothyroidism is poorly identified. Biochemical thyroid assessment is a simple test that can be incorporated into the assessment of the surgical patient. In modern practice it is important to consider the cost benefit ratio before performing additionally investigations on patients. Although significant surgical complications of hypothyroidism are rare it is difficult to discern what proportion of patients' symptoms may be affected by low levels of free T4.

Compliance of all medications, including thyroxine, is a well-known phenomenon throughout the world of medicine. Studies have shown states that only $50 \%$ of patients with chronic conditions comply with their treatment regime (Sabaté, 2003). Reasons for non-compliance with treatment are multifactorial, often including side effect profiles and lack of understanding of the benefits of taking the medication (Ngoh, 2009). In many cases these issues can be resolved by in depth discussions of advantages and disadvantages with the patient, and including them in the decision making process (Conn et al., 2016).

An aspect of long-term therapy that can be overlooked is managing the most commonly encountered side effects whilst on essential medications such thyroxine, anti-epileptics and analgesia. In the setting of chronic pain managed with opioids all clinicians are acute aware of the need for anti-emetics and laxatives in conjunction with the morphine derivative to obtain an effective balance of analgesia and unwanted symptoms (McNicol et al., 2003). However, constipation and subtherapeutic thyroxine treatment is not as commonly encountered and is therefore not at the forefront of concerns for this subset of patients.

After a decade of taking her thyroxine this lady made an active decision to stop her thyroxine. The patient perceived that cessation of thyroxine treatment resulted in improved symptom control of her irritable bowel syndrome. Despite general 
practitioners and consultants challenging this health belief the patient remained non-compliant. This lack of compliance was echoed biochemically with TSH levels $>100$ recorded in 2012 and on admission to hospital in 2016, despite the patient's assertion that she was taking regular thyroxine.

Improving compliance is both time-intensive and multifaceted. Evidence based techniques include acceptance of the need for compliance, patient's understanding of the need for daily medication and regular contact with health professionals for on-going negotiations with regards to the treatment and its impact on the patient's lifestyle (Winnick et al., 2005).

In conclusion this clinical encounter stresses the vital importance of communication in the setting of compliance and adherence of chronic conditions and long-term medications. It highlights that surgeons must not ignore or overlook potential medical conditions that may contribute to the numbers and complexities of surgical admissions. A more open view of the medical complexities of the surgical patient may prevent life threatening surgical complications such as perforation.

\section{References}

Bergeron, E., Mitchell, A., Heyen, F., Dubé, S. (1997) Acute colonic surgery and unrecognized hypothyroidism: a warning. Dis. Colon Rectum 40, 859-861.

Canaris, G. J., Manowitz, N. R., Mayor, G., Ridgway, E. C. (2000) The Colorado thyroid disease prevalence study. Arch. Intern. Med. 160, 526-534.

Chistiakov, D. A. (2005) Immunogenetics of Hashimoto's thyroiditis. J. Autoimmune Dis. 2, 1.

Conn, V. S., Ruppar, T. M., Cooper, P. S. (2016) Patient-centered outcomes of medication adherence interventions: systematic review and meta-analysis. Value Health 19, 277-285.

Ebert, E. C. (2010) The thyroid and the gut. J. Clin. Gastroenterol. 44, 402-406.

Liu, L., Hua, F. Z., Li, Q., Wang, K., Shao, J. H. (2011) Surgery for complicated diverticular disease: primary or secondary anastomosis after colonic resection. Cochrane Database Syst. Rev. 2011(12), CD006141.

McNicol, E., Horowicz-Mehler, N., Fisk, R. A., Bennett, K., Gialeli-Goudas, M., Chew, P.W., Lau, J., Carr, D. (2003) Management of opioid side effects in cancer-related and chronic noncancer pain: a systematic review. J. Pain 4, 231-256.

Müller-Lissner, S. A., Kamm, M. A., Scarpignato, C., Wald, A. (2005) Myths and misconceptions about chronic constipation. Am. J. Gastroenterol. 100, 232-242.

Ngoh, L. N. (2009) Health literacy: A barrier to pharmacist-patient communication and medication adherence. Pharm. Today 15, 45-57.

Pares, D., Biondo, S., Miró, M., Fraccalvieri, D., Julia, D., Frago, R., García-Ruiz, A., Martí-Ragué, J. (2005) Results and prognostic factors in the Hartmann procedure. Cir. Esp. 77, 127-131.

Sabaté, E. (2003) Adherence to Long-term Therapies: Evidence for Action. World Health Organization, Geneva.

Winnick, S., Lucas, D. O., Hartman, A. L., Toll, D. (2005) How do you improve compliance? Pediatrics 115, e718-e724.

Zachariah, S. K., Raja, N. (2010) Spontaneous perforation of the colon and hypothyroidism: report of a case and review of literature. Gastroenterology Res. 3, 147. 